EPJ Web of Conferences 92,02096 (2015)

DOI: $10.1051 /$ epjconf/ 20159202096

C Owned by the authors, published by EDP Sciences, 2015

\title{
Induced fluid rotation and bistable fluidic turn-down valves (a survey)
}

\author{
Václav Tesar̆ ${ }^{1, a}$ \\ ${ }^{I}$ Institute of Thermomechanics v.v.i., Academy of Sciences of the Czech Republic \\ Dolejškova 5, 18200 Praha-Kobylisy, Czech Republic
}

\begin{abstract}
Paper surveys engineering applications of an unusual fluidic principle - momentum transfer through a relatively small communicating window into a vortex chamber, where the initially stationary fluid is put into rotation. The transfer is often by shear stress acting in the window plane, but may be enhanced and perhaps even dominated by fluid flow crossing the boundary. The case of zero-time-mean fluid transport through the window has found use in experimental fluid mechanics: non-invasive measurement of wall shear stress on objects by evaluating the induced rotation in the vortex chamber. The case with the non-zero flow through the interface became the starting point in development of fluidic valves combining two otherwise mutually incompatible properties: bistability and flow turning down.
\end{abstract}

\section{Introduction}

In contrast to classical hydraulics and pneumatics which operate by means of mechanical moving or deformed components like pistons, slide valves, membranes, bellows, and similar objects - fluidics in its pure form handles fluid by clever use of aerodynamic and hydrodynamic phenomena in constant-geometry cavities. For this purpose it was essential to find and develop unusual fluid flow interaction phenomena, some of them not discussed in earlier literature.

The most popular effect utilised in the no-movingpart fluidic devices is inertia of fluid jets, generated by flow from a nozzle. This is used in no-moving-part diverter valves with control of the jet flow direction by an action of another, much weaker control input flow from a control nozzle (or more nozzles). The large difference between the weak control flow and strong output means the devices posses an amplification property. The basic jet-deflection mechanism has been developed into a wide spectrum of various designs [1]. Often used is the Coanda effect of jet attachment to an adjacent wall. This makes possible maintaining the jet deflected even after the control action has ceased. With two different attachment walls the devices are described as bistable: they can stay deflected in one of two regimes, requiring the control effect only for the brief moments of switching the jet flow from one regime to the other one.

While diverting effects are offered in a multitude of designs, choices of fluidic valve design are much more limited if the task to be realised is to turn the flow down. Only exceptionally this may be done by use of the "fluid plug" [2] principle. The better known turning-down mechanisms use centrifugal force acting on rotating fluid in a vortex chamber with central exit. When the fluid is put into rotation, the centrifugal force prevents it from reaching the exit (only a small amount of fluid gets there through the boundary layers where the rotation is not intensive enough) [3].

An interesting - but so far rarely used - principle utilisable in fluidics is generating rotational motion in the vortex chamber by transferring moment of momentum from another, neighbouring space through a small communicating window, Fig. 1. In the input chamber, at left in the picture, the fluid moves (the illustration shows

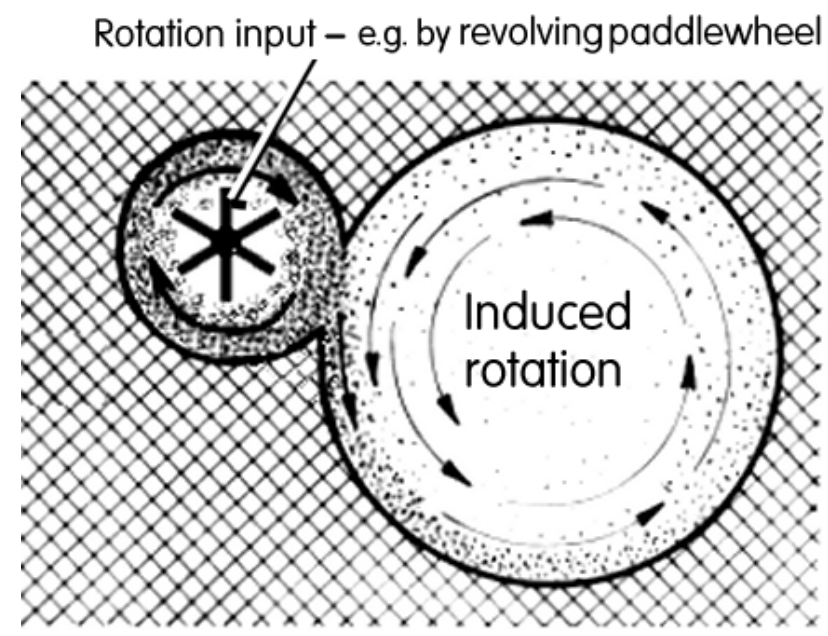

Figure 1. A small window communicating adjacent chambers suffices for transfer of momentum from rotating fluid in the input chamber (at left) to the initially stationary fluid in the chamber at right.

\footnotetext{
a Corresponding author: tesar@it.cas.cz
} 


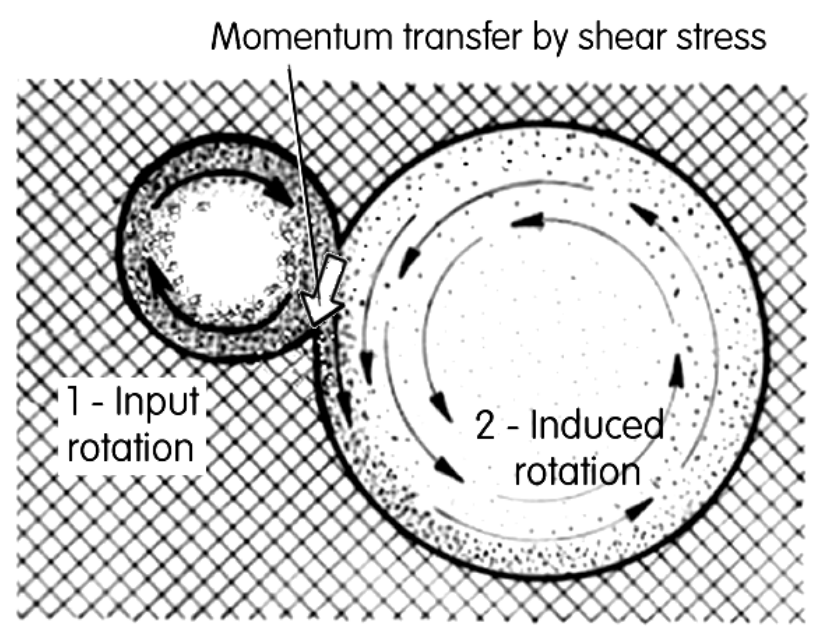

Figure 2. General features of the momentum transfer into the driven vortex chamber by shear stress acting in the window Sometimes (dependent upon the window geometry and availability of a source and sink) the transfer also involves flow of some momentum-carrying fluid through the window.

it kept in rotation by a paddlewheel). In the vortex chamber the fluid is initially stationary and the transfer of momentum puts it into the induces rotation. The main mechanism of the transfer is the shear stress acting in the window, Figs. 2 and 3. In laminar regime - for which is valid the Newton's law in Fig. 3 - the shear stress is a not very powerful mechanism. The transfer to the inducedrotation cavity becomes much more efficient if the primary motion is turbulent, especially if the downstream edge of the window is able to "peel-off" some of the turbulent eddies and move them through the window. It may be even possible to get a more efficient transfer by a momentum-carrying fluid flowing at a non-zero timemean rate through the window into the secondary chamber space, provided an overall balance may be established by similar return transgressing at the other, upstream edge of the window. If there is a source in the input primary chamber and a corresponding sink in the induced-rotation secondary chamber, the non-zero timemean flow rate increases in importance as the mechanism

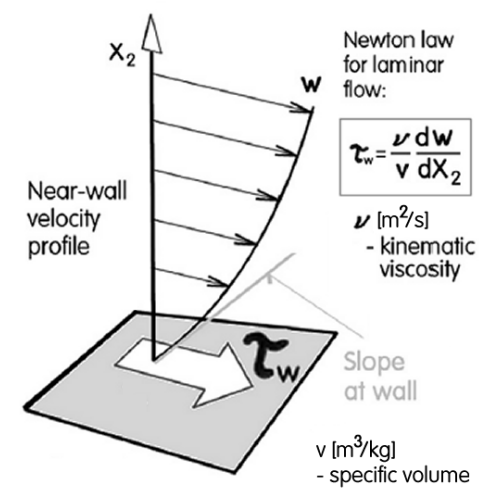

Figure 3. The main force action in the transfer window without mass flow rate through the boundary is the effect of the shear stress $\tau_{\mathrm{w}}$. Its magnitude is more or less identical to that in the input-chamber wall upstream from the window. of the transport - and in the extreme case (which may no more belong into the discussed category of flows) the flow rate across the window boundary may become the dominant transfer effect.

\section{Induced rotation and its measurement}

The shear stress, denoted $\tau\left[\mathrm{Pa}=\mathrm{N} / \mathrm{m}^{2}\right]$ is defined as the component of stress in fluid coplanar with the investigated surface (while the normal stress acts on this surface in the perpendicular direction).

Of particular importance [4] is the wall shear stress $\tau_{w}$. This is the extrapolated value obtained as one progresses in the perpendicular direction towards the solid surface past which the fluid flows. Wall shear stress has its important established role as reference value especially in studies of turbulent boundary layers, where it often enters the expressions as the corresponding value of friction velocity $W_{\tau}[\mathrm{m} / \mathrm{s}]$

$$
w_{\tau}=\sqrt{\tau_{w} v}
$$

This quantity is used for evaluating the near-wall conditions in turbulent flows. It is not without use even in laminar flows (after all, in turbulent flows the presence of the wall suppresses the vortical motion so that at the wall there is a sub-layer known to have properties governed by laminar law - though the conditions there are not really steady).

As far as the practical engineering importance is concerned, the wall stear stress is the essential quantity for evaluation of drag of bodies (ship hulls, aeroplane fuselage, ...) moving relative to surrounding stationary fluid. The aerodynamic drag consists of two components. The first one is the pressure drag, which is evaluated by integrating over the body surface of local pressure force components acting in the direction parallel to the flow. The other, second component is the friction drag evaluated by integration of wall shear stress force components parallel to the trajectory of investigated motion.

The importance of the wall shear stress is reflected in the magnitude of effort devoted in Fluid Mechanics to its experimental evaluations. This is a task that is by no means easy. Indeed, in the classical textbook by Thwaites [5] was said: "...to measure accurately skin friction is a perpetual challenge to experimenters." Historically, the earliest wall shear stress measurements were made on flat plates moved at known velocity in water with the intention to obtain knowledge useful for shipbuilding. The first data obtained this way were poublished by W. Froude in 1862. This measured total drag force could not be simply corellated with the plate dimensions and it was soon realised that really needed are local data at particular points on the surface. Only much later could be the local shear stress measured by probes positioned on the investigated wall. Early probes were piezometric, i.e. generating at their output a difference between two pressure values (usuallymeasured by a micromanometer). Typical examples of these probes are, e.g. Stanton tube, Preston tube, or the sub-layer fence - discussed in ref. [6]. The main problem encountered was that the the 


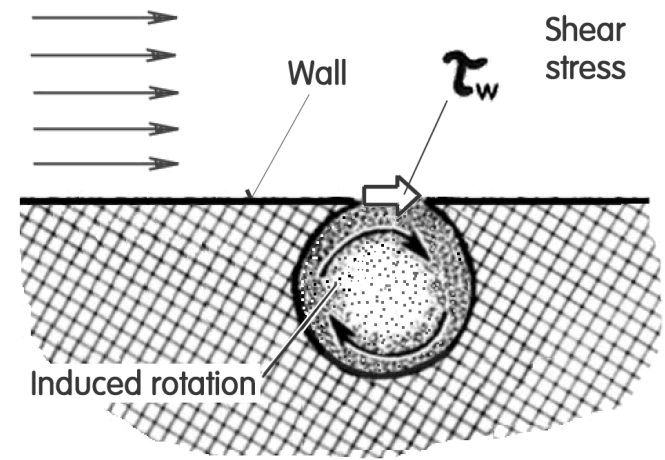

Figure 4. Wall shear stress $\tau_{\mathrm{w}}$ on the surface exposed to fluid flow is a quantity of essential importance in fluid mechanics and its application - its integral over the surface area determines the frictional component of the aerodynamic drag. Present author's idea is to measure $\tau_{\mathrm{w}}$ by evaluating the induced rotation. The advantage is no disturbing effect on the investigated outer flow.

presence of these probes, even if they were made as small as possible, changes the investigated flowfield sometime quite significantly. For example, the large difference in magnitudes of laminar and turbulent wall shear stress is often used to identify the location where laminar/turbulent transition takes place. Unfortunately, the presence even of only millimetre-sized Preson tube was demostrated to cause an earlier transition. There are even flows - a typical case being the Coanda-effect of jet attachment to a curved wall - in which the presence of the probe was demonstrated to cause separation of the flow from the attachment surface - and hence a substantial change of the whole investigated flowfield.

In an attempt to avoid these problems, many contemporary shear stress measuring experimenters utilise the existing analogy between momentum and heat transfer - the latter being relatively easy to evaluate due to the progress of electronics. Typical set-up is to provide the surface in the selected location with a thin conductive film and heat it by passage of current - often by using for the purpose the electronic circuitry of hotwire anemometric systems. The cooling effect of the fluid flow is related to the local shear stress in the fluid. There is a general belief that if the conductive film is thin enough (not projecting significantly above the wall) this method causes no disturbance to the investigated flowfield. Unfortunately, this belief is wrong. As was demonstrated in [7], the heating the surface by mere 20 ${ }^{\circ} \mathrm{C}$ can change the flowfield beyond recognition, especially on curved wall surfaces, such as the flow with the Coanda-effect attachment.

\section{Use in measuring wall shear stress}

A solution without the flow-influencing drawbacks is offered by the induced-rotation phenomenon. The basic idea is presented in figure 3 . The primary chamber of Fig. 2 is here replaced by the investigated large-scale flowfield. The wall at which the shear stress is to be measured is provided with a hollow cavity - a small axisymmetric vortex chamber, with a part of its circumference opened through a small momentumtransfer window. Obviously, there is nothing protruding from the wall surface and since the window has a small length in the flow direction, the flow is practically not influenced. Of course, theoretically some small amount of energy is necessarily extracted form the measured flow to drive the induced rotation, but this experimental evidence supports the fact that this amount is negligible. In ref. [15] is presented a convincing demonstration that even in the case of a flowfield extremely sensitive to the presence of tiny objects on the wall the difference in velocity fields with and without the induced-motion

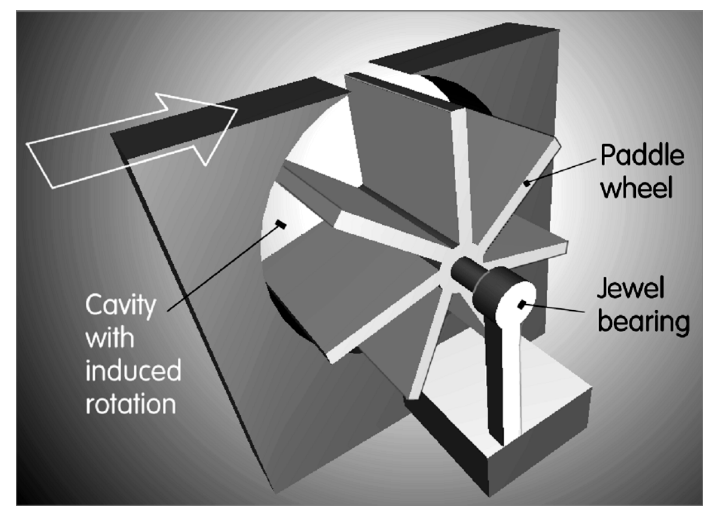

Figure 5. Evaluation of the wall shear stress $\tau_{\mathrm{w}}$ on the surface exposed to fluid flow by counting passages of light between the paddles per unit of time. This use of as small paddlewheel in the induced-rotation cavity was based upon present author's suggestion and realised by Dr. Skrzynski, Warsaw University of Technology (using bearings from a mechanical watch).

cavity is not recognisable. It is a proof that the separation and laminar/turbulent transition phenomena are not influenced. Because of the small window length in the flow direction, the shear stress value there is practically identical in magnitude to the wall shear stress immediately upstream. The advantage is the intensity of fluid rotation inside the vortex chamber may be easily and directly measured.

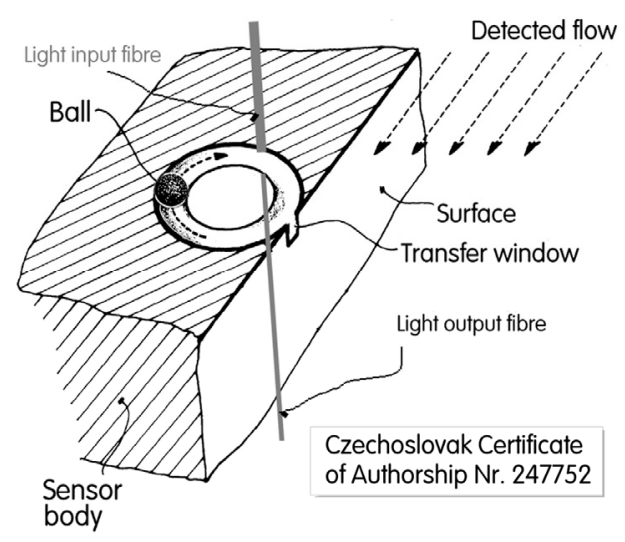

Figure 6. Wall shear stress may be $\tau_{\mathrm{ww}}$ measured by counting the interruptions of light in the light-conducting fibres by small polystyrene ball running in a semi-toroidal raceway [10]. 


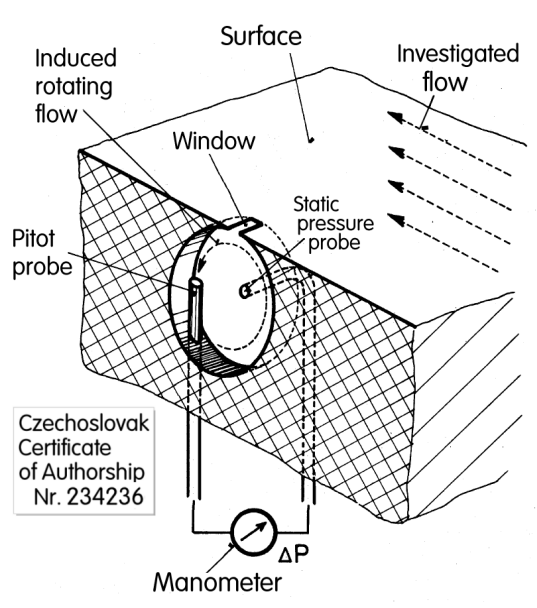

Figure 7. Another wall shear stress measurement setup, using capillaries (from hypodermic needles) for the Pitot and static probes [11].

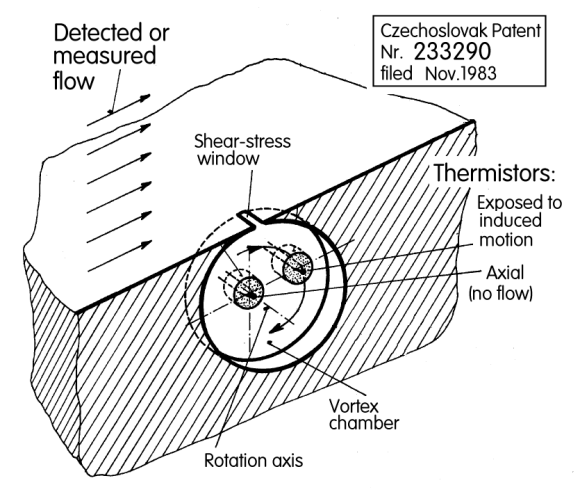

Figure 8. Yet another shear-stress measurement with induced rotation - by differential cooling of thermistor pair [12].

Several methods of measuring the intensity of induced rotation were investigated by the present author and are described in [13]. Some of the tested configurations are subjects of Czechoslovak Patents (or earlier Certificates of Authorship), but all of these documents have in the meantime expired. Thus there is nowadays no reason for fear of possible legal restrictions. One of the first embodiments of the shear-stress measurement principle was made by Dr. Skrzynski, Fig. 5. Another typical example of the induced-rotation measurement method, from Certificate of Authorship [10], is presented in Figure 6. All that is visible from outside on the wall is the small window, with dimensions that may be as small as mere tenths of a millimetre. Below the window, inside the wall under the surface is in the location of measurement a small vortex chamber. In the case from Fig. 6 it has on its circumference a raceway - a semitoroidal groove - in which there is a very light small polystyrene ball, free to roll. The induced rotation forces it to continuous rolling run inside the groove. The energy needed to put it in motion is extremely small. The speed of the ball running was in the experimental verification easily measured by counting the interruptions of the light between the ends of light fibres (Figure 4). Other alternatives, without moving components, and therefore more resistant to external acceleration, are presented in

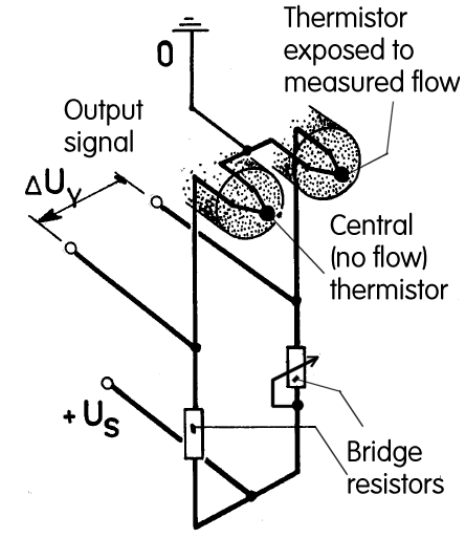

Figure 9. Simple electric bridge circuit used with the thermistors of Fig. 8 - from ref. [12].

Figs. 7 and 8. Practical advantage of the approach shown in Fig. 7 was in availability of a micromanometer, the basic instrument in any aerodynamic laboratory. The other example, presented in Fig. 8, uses the pair of thermistors, connected into a balanced Wheatstone bridge Fig. 9, measures the induced rotation by the voltage difference between the thermistor positioned at different radii from the rotation centre.

\section{The "submerged fence" sensor}

It is generally not fully appreciated that the classical piezometric skin friction sensors - such as the wellknown Stanton, Preston, or fence probes - are all actually also based on measuring the intensity of induced rotation. It is a vortical rotation driven by the shear stress on the outer boundary. This fact, however, is not very obvious and, as a result, the geometry of the cavities is normally not designed to provide favourable condition for the stationary vortices (which may be in some configurations

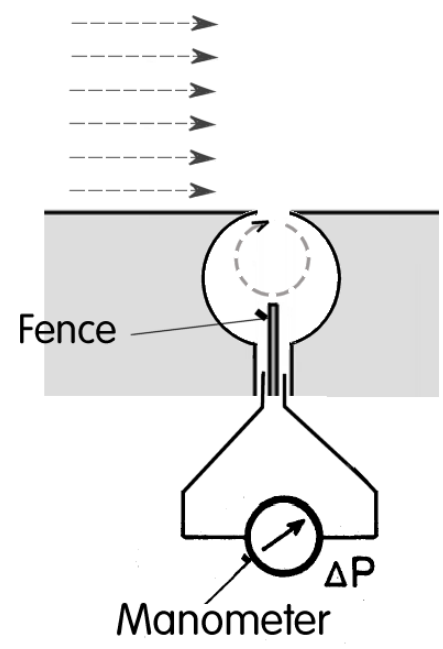

Figure 10. The induced rotation, driven by the investigated flow, can withstand a quite strong opposition from various components causing hydrodynamic loss in the induced-rotation chamber. Here such component is the fence which generates large measured pressure difference - without influencing in a significant measure the investigated flow. 


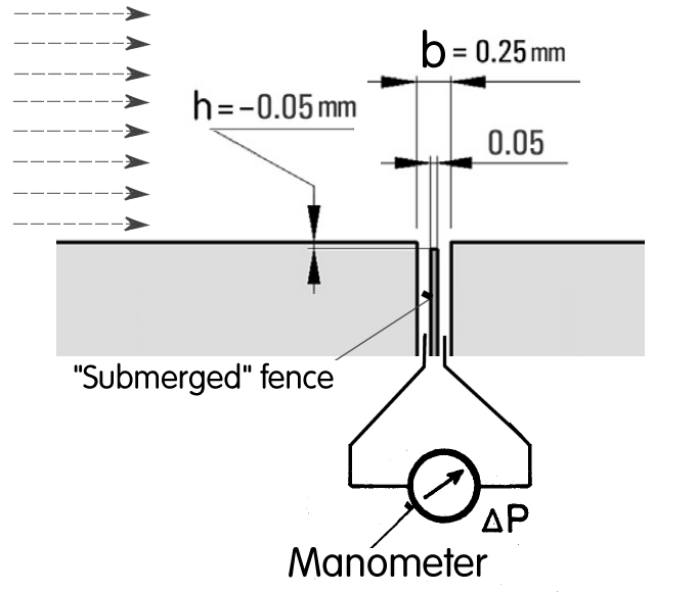

Figure 11. Wall shear stress measurement with the "submerged" fence [13]. Also in this case is actually used the induced rotations. Not knowing the fact, vortex cavity shapes chosen because of simplicity and not particularly suitable for the role are used.

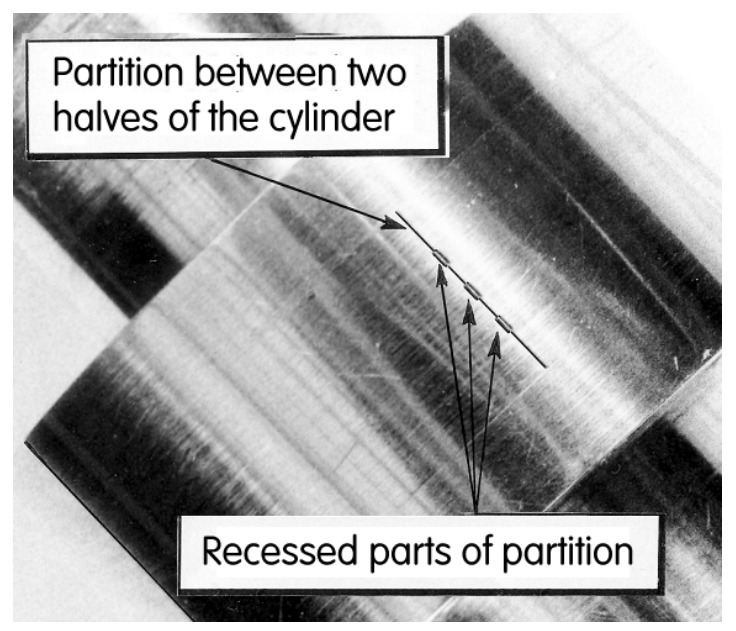

Figure 12. Photograph of the sensors for shear stress measurement in tangential flow past cylindrical surface. The surface consist of two halves (upstream and downstream one), the partition separating them is locally recessed in the slits to form the "submerged fence".

actually exposed to the surrounding flowfield so much that they are more or less regularly shed - and, of course, inputting energy into the vortex that is lost from the sensor by being shed decreases the energetic efficiency of the method). An example demonstrating the stationary vortices is presented in figures 7 and 8 . This case is actually a little-known sensor configuration, related to the better-known case of the "sublayer fence". The fence is a thin rectangular component (a piece of sheet metal) positioned between the two channels which carry the pressure signals into a micromanometer (the latter is, as usual, of the differential measurement character). In the standard case $h>0$, the fence protrudes to the height $h$ above the wall past which flows the fluid. This obstacle, of course, causes a higher pressure in the upstream channel and a lower pressure in the downstream one.

The problem with the fence - same as with almost all piezometric (pressure measuring) methods - is the

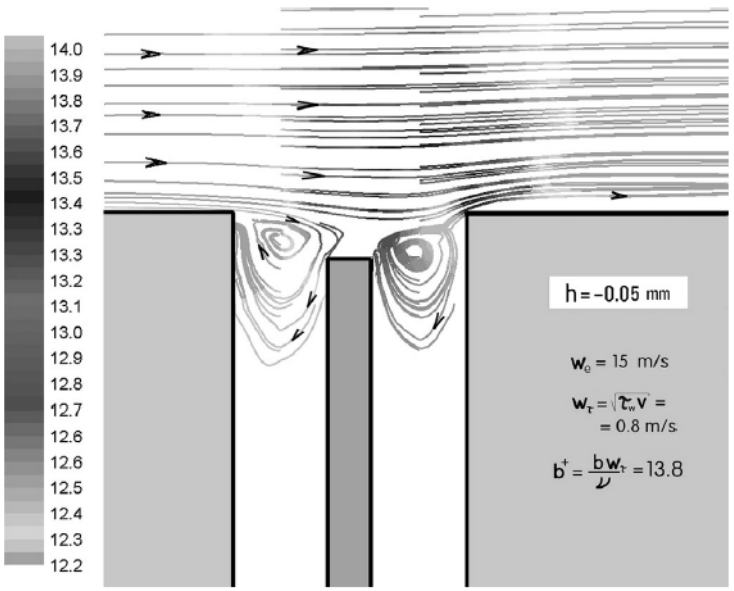

Figure 13. Computed pathlines of the flow in the small spaces vicinity of the top of "submerged fence" (Fig 11). Somewhat enigmatically, the operation depends on creation of two stationary vortices $[15,16]$.

influencing of the measured flowfield by the presence of the probe. This is particularly unpleasant in such sensitive flows like the low Reynolds number Coanda-effect attachment to a curved wall: the fence can cause premature transition into turbulence and even premature separation of the jet flow from the attachment wall. The problem was solved by the present author as long ago as in 1974, when it was discovered [8] that the fence retains its measuring capability even if it is moved below the surrounding surface, i. e for $\mathrm{h}<0$.

The resultant non-invasive "submerged fence" method [8, 15] was recently used in a number of exceptionally demanding experimental applications and found suitable (in some cases, indeed, the only actually suitable) solution of the shear stress measurement problem [14]. Figure 7 presents the basic dimensions of the used sensing slits while the computed pathlines are presented in Figure 8. They show inside the available small space in the narrow slits that form the sensor actually two induced rotational motions, one in front of and the other one behind the fence.

Since the presence of these vortices - which was proved in [14] to be the necessary condition for the sensor generating a useful output signal - was not known, the elongated and rectangular shape of the cavities in which the vortices rotate are not particularly suitable for this particular role and were simply chosen so as to ensure the simplest manufacturing. The following Figure 9 shows how the sensor may be adapted to provide better conditions for the generation of the induced rotation. There are semi-toroidal grooves both in front of the fence and behind it inside the wall. Together they form an annular space inside which the induced rotation may be more intensive and less prone to viscous damping. Also - since in this configuration the fence between the two pressure output signal leads need not be submerged and may be protrude from its surrounding walls - this version makes the generated output pressure difference larger. This may be particularly useful in determining the flow separation location, which is defined by the shear stress there approaching zero value. 


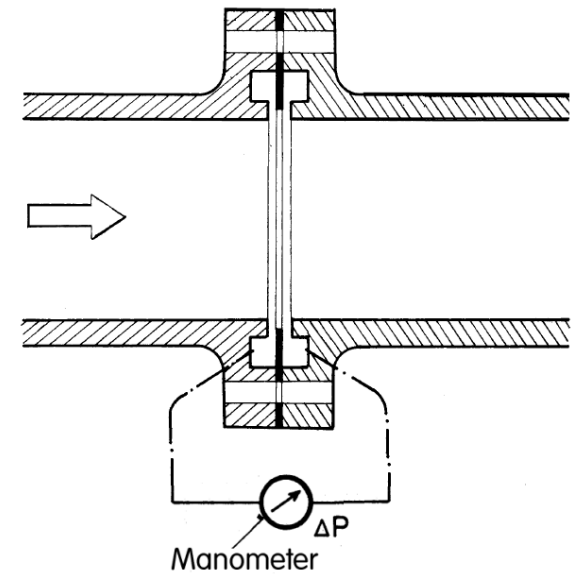

Figure 14. Measuring flow rate in a pipeline according to [17] using the idea of submerged fence. Contrary to the usual orifice plate of traditional flowmeters, the layout brings the advantage of no detectable hydraulic losses.

An interesting extension of the "submerged fence" idea is presented in Figure 10. In that case the fence occupies the whole circumference of a pipe cross section. The shear stress evaluated from the measured pressure difference is here not the final quantity of interest. This is here the flow rate in the pipe (of course associated with the pressure difference in a unique manner). The configuration described in [9] is very similar to the standard measurement with an orifice. The advantage over that classical setup is obvious: decreased hydrodynamic loss since there is no obstacle to the flow and hence increased efficiency of fluid transport in the pipeline. A slight disadvantage is the lower measured pressure difference - which is no real problem in the contemporary era of precise digital micromanometers.

\section{Devices with flow through the boundary in the window}

The first engineering application of the authors's idea of induced rotation in the version with fluid flow through the boundary was in cyclonic separation of particulate material by Prof. Syred in 1992. Usual cyclone layouts give little consideration to pressure recovery. Kinetic energy of fast swirling motion is dissipated by turbulence and friction. Tangential off-take discussed in [17] with associted diffuser could significantly improve energy consumption of the separator. The idea was later further developed in the popular vacuum cleaners according to the Patent [18].

A configuration which is essentially the reverse of the above off-take idea is generating the rotation in the pre-chamber (Fig. 15) by a nozzle flow and transfer it into the main vortex chamber where it is possible to use the centrifugal forces acting on the rotating fluid to control the flow rate passing through the device. Importance of this case is due to the fact that the resultant no-moving-part valve device can serve for flow control with unique properties. The illustrations in Figs. 15 and 16 presents an example, with rotation in pre-chamber transferred into the vortex chamber at right. The entrance for the fluid is at left and next to it there are perpendicularly oriented control nozzles, dictating the rotation sense. A small control flow pulse applied in one of these nozzles can switch the incoming large flow.

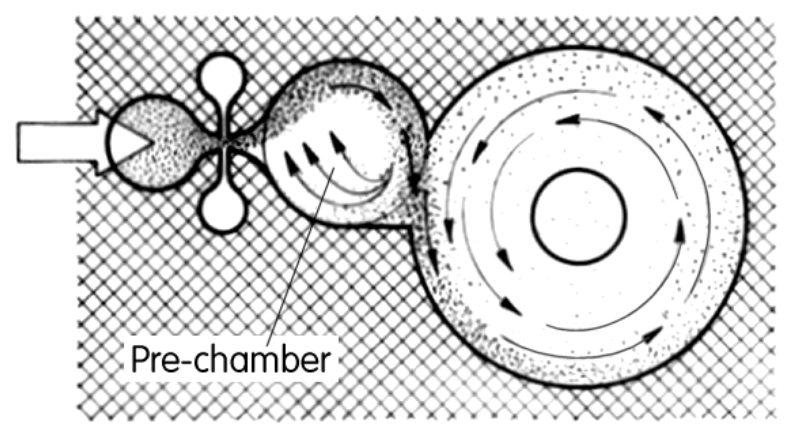

Figure 15. The basic principle of the no-moving-part fluidic valves combining otherwise mutually excusive bistability and capability to turn the flow down - using non-zero flow rate through the window $[19,20]$. Shown here is the regime of clockwise rotation in pre-chamber.

The situation in Fig. 15 shows the resultant counterclockwise rotation generated inside the large main chamber caused by the fluid entry. This large chamber has an outlet in its centre, allowing the fluid to enter this chamber through the window. The flow through the device, however, in the regime of Fig. 15 is small. Fluid flow towards the central exit is opposed by the centrifugal force acting on the rotating fluid. To conserve the moment of momentum inside the large vortex chamber, the fluid increases its tangential velocity as the radius of rotation decreased on its progress towards the exit.

If the configuration of the communicating window between the two chambers were symmetric relative to the line connecting their centres, all that such switching could do would be only the change the sense of rotation. The geometry suggested in Figs. 15 and 16, however, is more interesting. One of the edges of the windows in the pre-chamber is shaped so that it directs the fluid flow into the large chamber radially, towards the central outlet. This means that in the regime of anti-clockwise rotation in the pre-chamber - Fig. 16 - the fluid flow passes through the window radially. As a consequence, there is no centrifugal effect opposing the flow. The overall pressure difference across the device is much lower than in the regime with rotation. This is the turning-down capability. At the same time the device exhibits the bistability: it can stay stably (i.e. re- turning back after a small disturbance) in either one of the two regimes. In author's experiments, the two control inlets were connected (in a similar manner as in [19]) by a feed-back loop tube. This resulted in the device operating as a selfexcited fluidic oscillator [20].

In Fig. 17 is presented a photograph of the laboratory model that was used in the oscillator role switching periodically between the "closed" (Fig. 15) and "open" (Fig. 16) regimes (in the latter regime, of course, the flow through the device is not entirely stopped - some 
fluid passes through the boundary layers of the walls, where the centrifugal force is weaker - but only turned down, which in most applications suffices).

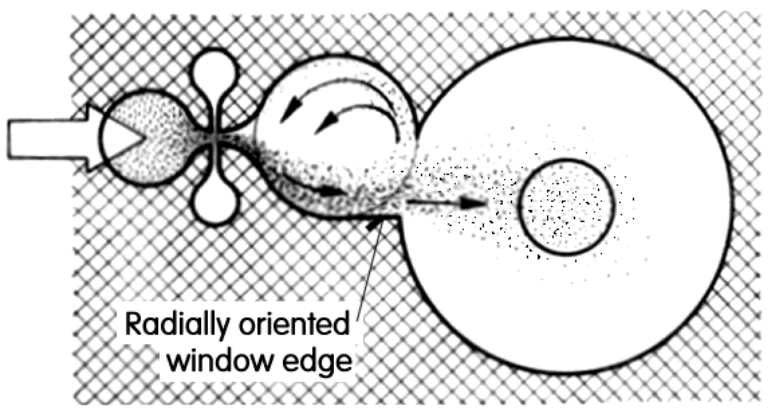

Figure 16. Shaping of walls near the communicating window generates, compared with Fig. 15, in the anti-clockwise prechamber rotation regime a radial flow leading to low overall hydraulic resistance (because of no need to overcome the centrifugal effect).

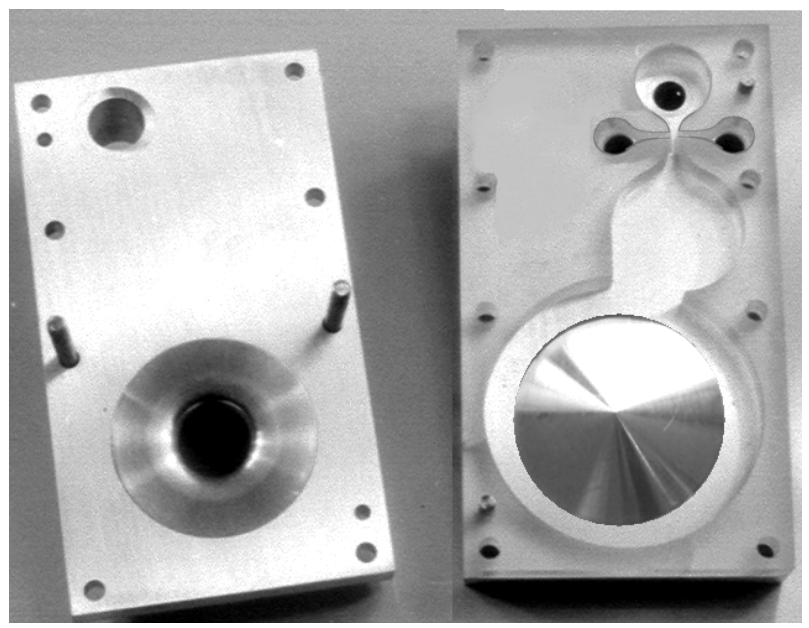

Figure 17. Photograph of the laboratory model of the valve used to investigate the operation in the two alternative regimes as shown in Figs 15 and 16.

The laboratory tests with the model shown in Fig. 17 revealed necessity of changing some parameters. One of these changes was providing more suitable shape of the Coanda-effect attachment walls immediately downstream from the control nozzles - the walls in Fig. 17 were too short and of too small curvature radii, making the attachment insufficient.

On the basis of the positive experience with the model, the author has later built a substantially scaled-up model, the photograph of which is presented in Fig. 18 (with young lady serving as the scale reference) and cross-section drawing in Fig. 19. It was a fluidic bistable turning-down valve, the main component of terminal units of air-conditioning systems. The units were to be placed into the ceiling of the conditioned room. The large scale - and consequent slow velocities - were needed to suppress the aerodynamic noise. In place of a simple hole exit from the main vortex chamber, the terminal unit was provided with radial (or, more precisely, diagonal) diffuser by means of the component $\mathrm{k}$ - to slow down the air exit velocity.

The device with the Coanda-effect attachment walls was, of course, capable of operating in only two regimes. To use it in the conditioning system that required continuous variation of the air flow rate, the valve was operated in the PWM control regime - with variable length of the period during which the valve was in the "open" regime. This is a particularly good example of the usefulness of the PWM principle in fluidics. The radial jets of cold air generated at the exit from the diagonal diffuser were requested to reach the whole length of the conditioned room in the summer regime. Being cold (and therefore heavier) the jets possess the natural tendency to

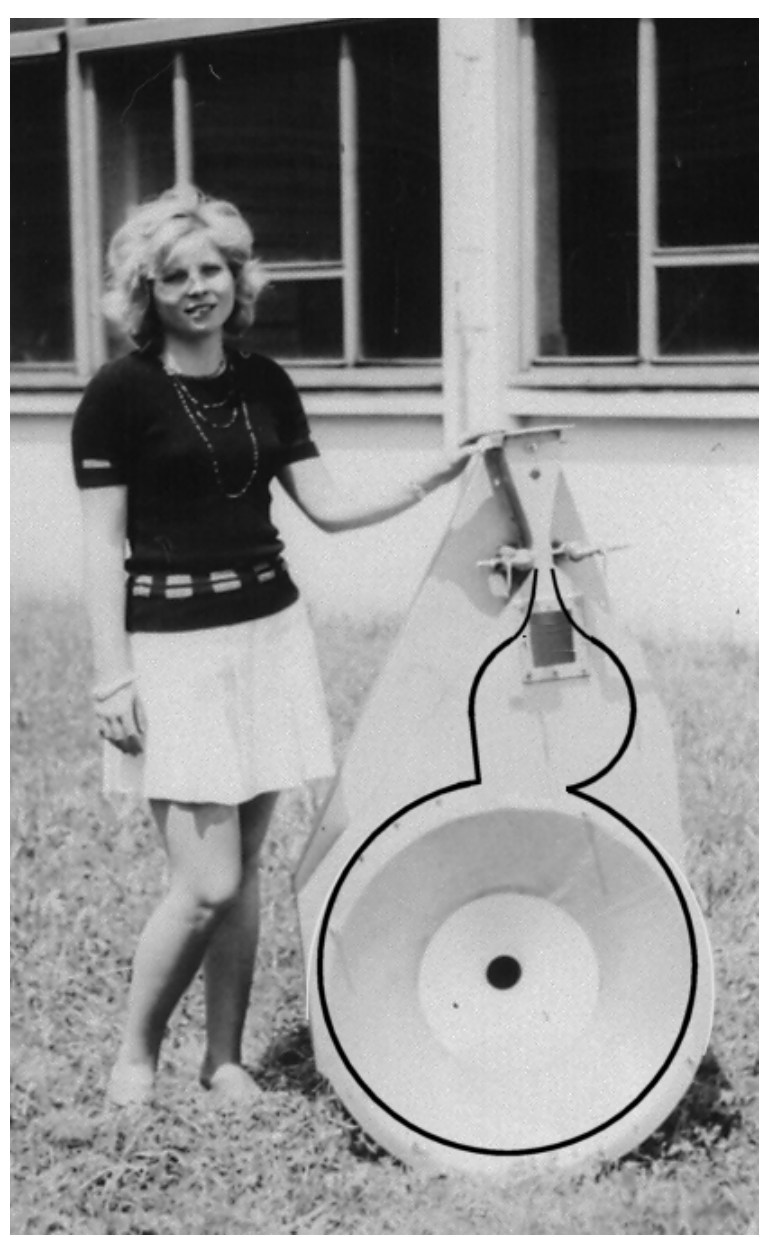

Figure 18. Photograph of the full-scale test model of the valve shown in Fig. 17. Contours of the internal cavities are here indicated by hand-drawn heavy line. The cone k (Figure 19) that defined the exit diagonal diffuser is here removed.

stop following room ceiling and fall down to the floor. This is prevented by the jet being given sufficient momentum in the terminal unit exit. If the room temperature were controlled by the standard method of decreasing the jet velocity, the momentum at the small flow rate situation would not suffice. In the PWM regime, however, the jet momentum remains constant throughout the whole "open" flow pulse; the room 


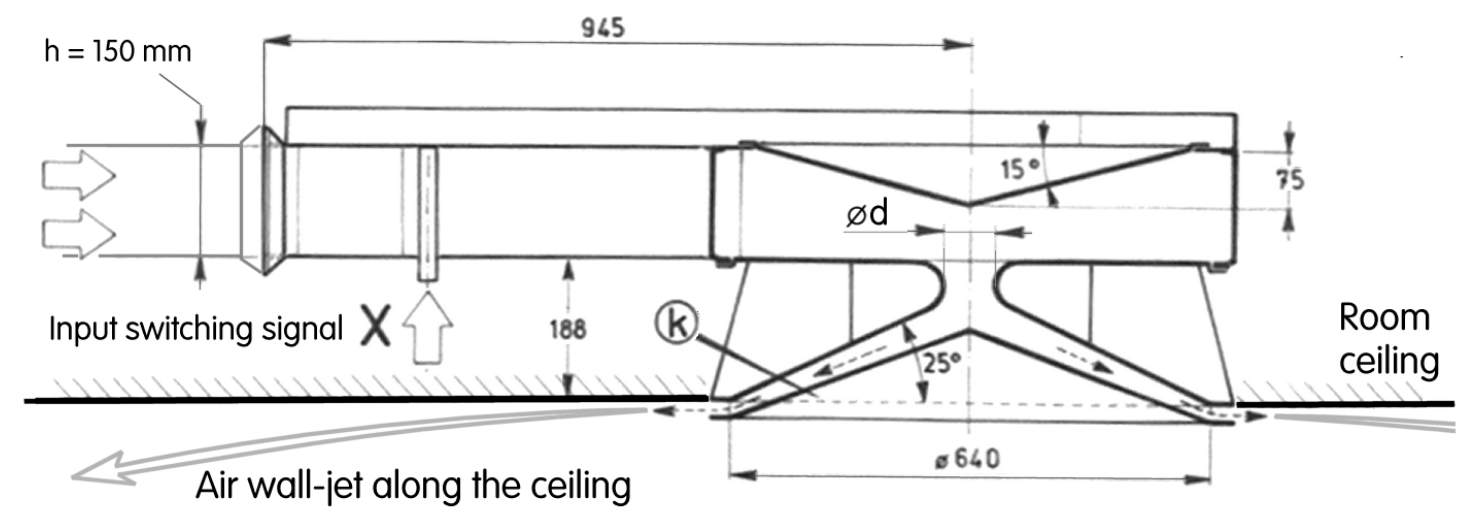

Figure 19. Drawing of the terminal unit of an air-conditioning system with pulse-width modulation of the exit flow. The configuration corresponds to Figs. 15 to 17. Large scale was dictated by the request for low velocity (to avoid aerodynamic noise).

temperature is controlled solely by varying the pulse duration.

It may be useful to compare the different relative sized of the central exit hole from the main vortex chamber - the diameter $d$ in Fig. 19. In the small model presented in Fig. 17 (in the top cover plate, shown in the left-hand part of the photograph) the relative diameter of the exit is large — while the experience gained during the investigations has led to preference given to the diameter much smaller. This is visible in the large-scale version photograph in Fig. 18.

Indeed, performance of the model - measured by the ratio q of dissipances Qclosed in the "closed" and Qopen in the "open" regimes (dissipance is defined e.g. in eq.(1) of reference [24]) - was found to depend much on the diameter $\mathrm{d}$ of the central exit from the vortex chamber. To gain information about this dependence, author performed a series of dissipance measurements with various exit diameters $d$. The rather complex (rounded and with the 25 deg inclination - Fig. 19) geometry of the exit would make this investigation rather expensive with a series of such exits. The measurements were also complicated by certain non-constancy of the dissipance values (dependence of $q$ on Reynolds number). To simplify the investigations, the tests were therefore performed with very simple exit geometry - a hole in a plat plate [22]. Admittedly, this simple shape - moreover without the diagonal diffuser - exhibited significantly worse performance, the maximum obtained value with the simple exit was near to only $q=6$. However, the aim of this investigation series was obtaining an insight into the optimisation problem. The results are presented in Fig. 20. The diameter of the (simplified) exit hole is on the horizontal co-ordinate and the performance parameter $\mathrm{q}$ is plotted on the vertical co-ordinate. The above mentioned small dependence of $q$ on the Reynolds number is visible from the near-optimum dependence on the mass flow rate. It was soon found that the optimum was near to the configuration with the cross-sectional area of the exit $F_{Y}=\pi d^{2} / 4$ and the area $b \times h$ of the supply nozzle are very neat to each other. The difference between the values of these two dominant (smallest) areas could easily be explained by the difference in the respective shapes (the smooth contoured nozzle on one hand and the sharp-edged hole in the vortex chamber exit on the other hand. The knowledge of this optimality condition was later developed to the rukle formulated for mutual optimisation of fluidic devices in series presented in [22] - which has later led to an even deeper optimum matching study [23].

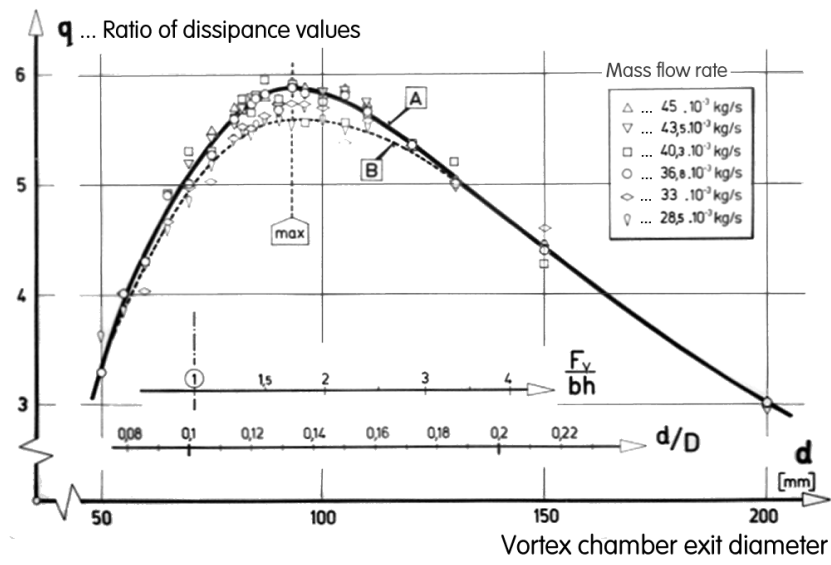

Figure 20. Performance (measured by the ratio of pressure drops in closed and open state) evaluated in tests with various diameters $d$ of the central exit (of simplified shape) from the vortex chamber Figure 19. Importance of matching the two dominant cross sections, $F_{Y}=\pi d^{2} / 4$ and $b \times h$ is obvious.

\section{Conclusions}

This survey paper is an attempt to bring to the attention of Fluid Mechanics community a number of engineering applications based on the induced-rotation idea which remains so far practically unknown and yet might be interesting.

\section{Acknowledgements}

This study of new or little-known principles and theory used in fluidics was supported by research grant Nr. 13-23046S obtained from GAČR - the Czech Science Foundation. Author also received institutional support RVO:61388998. 


\section{References}

1. S. Raghu, Experiments in Fluids, 54, 1455 (2013)

2. V. Tesař, Journal of Visualization, 6, 77, (2003)

3. C. F. King, Trans ASME - Journal of Dynamic Systems, Measurement and Control, 190, 44, (1987)

4. M. A. Day, Erkenntnis, 22, 285 (1990)

5. B. Thwaites, Incompressible Aerodynamics, Dover Publication (1987)

6. K.G. Winter, Progress in Aerospace Sciences, 18, 1 (1979)

7. V. Tesař, Control of flow separation by temperature gradient (Proc. of Euromech Colloq. 377 Praha, Czechoslovakia May 1998)

8. V.Tesar̆, Proceedings of theVth 'Jablonna' Fluidics Conf. Budapest, Hungary (1974)

9. V. Tesař, Czechoslovak Certificate of Authorship Nr. 169682, DOI: 10.13140/2.1.2266.5600, Jan 1975

10. V. Tesar̆, Czechoslovak Certificate of Authorship Nr.247752 DOI 10.13140/2.1.1113.7284, Mar. 1983

11. V. Tesar̆, Czechoslovak Certificate of Authorshp Nr. 234236, DOI: 10.13140/2.1.4259.4562, Mar. 1983

12. V. Tesař, Czechoslovak Certificate of Authorship Nr. 233290, DOI: 10.13140/2.1.1182.8485 Nov. 1983

13. V. Tesař, Proc. of Conf. 'Application of Experimental methods', Starý Smokove, Slovakia (1983)

14. . Tesař, Proc. of Vth. 'Jablonna Fluidics Conference, Budapest, Hungary (1974)

15. V. Tesař, Flow Measurement and Instrumentation, 33, 228 (2013)

16. V.Tesar̆, Proceedings of Internat. Conf. on Engng. Mechanics, Svratka, Czech Republic (2012)

17. V. Tesař, Czechoslovak Certificate of Authorship Nr. 204745, DOI: 10.13140/2.1.5155.9688, Jul. 1979

18. T. O'Doherty, M. Biffin, N. Syred, Proceedings of the Institution of Mechanical Engineers, Part E.: Journal of Process Mechanical Engineering, 206, 99 (1992)

19. J. Dyson, S. C. Jupp, A. MacLeod, US Pat. 6482246 , Oct. 1999

20. V. Tesař, Proc. of the 9-th Internat. Fluidics Conference, Paper A-5, Jablonna, Poland (1982)

21 V. Tesař, K. Hemzal, Proc. of the Vth 'Jablonna' Fluidics Conf., Budapest, Hungary (1975)

22. V. Tesar̆, Proc. of Conf. "Riadenie tekutinových systémov 97", University of Žilina, Slovakia, ISBN 80-7100-452-9 (1997)

23. V. Tesař, Chemical Engineering Research and Design, 87, 817 (2009)

24. V. Tesař, Sensors and Actuators A: Physical, 199, $379(2013)$ 